[Article]

\title{
石墨烯分子振动模式因子群分析与密度泛函计算
}

\author{
刘 波 ${ }^{1}$ 孙红娟 ${ }^{2, *} \quad$ 彭同江 ${ }^{2}$
}

('西南科技大学理学院, 四川绵阳 $621010 ;{ }^{2}$ 西南科技大学矿物材料及应用研究所, 四川绵阳 621010)

\begin{abstract}
摘要：运用因子群分析法对石墨烯的分子振动模式进行了理论分析, 得到石墨烯的分子振动模式, 计算出各 振动模式的光谱特性. 对所建立的石墨烯晶体的布拉维单胞模型采用基于密度泛函理论的第一性原理进行分 子振动频率与模式的计算, 所得的振动模式数目以及各振动频率的光谱特性同因子群分析方法所得结论一致. 结合上述计算结果, 通过系统比较石墨与石墨烯之间的红外光谱和拉曼光谱的差别, 从理论上解释了具有 $D_{6 n}$ 对称的石墨烯的 $A_{2 u} 、 E_{14}$ 红外活性特征振动模式没有在红外光谱中出现的原因.
\end{abstract}

关键词: 石墨烯; 因子群; 振动模式; 第一性原理; 红外光谱; 拉曼光谱

中图分类号: 0641

\section{Factor Group Analysis of Molecular Vibrational Modes of Graphene and Density Functional Calculations}

\author{
LIU Bo $\quad$ SUN Hong-Juan ${ }^{2, *} \quad$ PENG Tong-Jiang ${ }^{2}$ \\ ('College of Science, Southwest University of Science and Technology, Mianyang 621010, Sichuan Province, P. R. China;

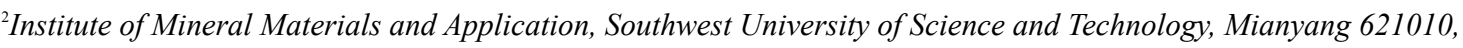

Sichuan Province, P. R. China)

\begin{abstract}
The molecular vibrational modes of graphene were analyzed theoretically by factor group analysis. The molecular vibrational modes of graphene and the spectral characteristics of each vibrational mode were calculated successfully. The molecular vibrational frequency and mode of graphene were also calculated by first-principles density functional theory based on establishment of the graphene Bravais crystal unit cell. The number of vibrational modes and corresponding vibrational frequency spectral properties calculated were consistent with the results obtained using factor group analysis. The above calculations and systematic comparison between the infrared and Raman spectra of graphene and graphite were used to determine why the infrared active vibrational modes $A_{2 u}$ and $E_{1 u}$ with $D_{6 h}$ point group did not appear on the experimental infrared spectrum of graphene.
\end{abstract}

Key Words: Graphene; Factor group; Vibrational mode; First-principles; Infrared spectrum;

Raman spectrum

\section{1 引 言}

2004 年, Geim 等 ${ }^{1}$ 利用微机械剥离法成功将石 墨剥离, 观察到具有稳定结构的二维单层石墨烯. 石墨烯的发现震撼了整个物理学界, 推翻了“热力 学涨落不允许二维晶体在有限温度下自由存在”的
认知. ${ }^{2,3}$ 单层厚度的石墨烯完善了整个碳族的结构 体系, 被认为是零维富勒烯、一维碳纳米管、三维石 墨的基本组成单元. ${ }^{4}$ 由于振动及声子性质的研究可 以推导出热导率和热容, 以及声子研究在准粒子动 力学及电子输运方面有着及其重要的作用, ${ }^{5}$ 因此石

Received: November 7, 2011; Revised: January 5, 2012; Published on Web: February 1, 2012.

"Corresponding author. Email: sunhongjuan@swust.edu.cn; Tel: +86-816-2419016.

The project was supported by the Doctoral Fund of Southwest University of Science and Technology, China (11ZX7135).

西南科技大学博士基金(11ZX7135)资助项目

(C) Editorial office of Acta Physico-Chimica Sinica 
墨烯的振动及声子性质引起了研究者的广泛兴趣. 梁维等 ${ }^{6}$ 基于晶格动力学理论, 采用力常数模型, 计 算了石墨纳米带的声子色散关系、振动模式密度和 比热, 结果表明石墨纳米带的声子谱特征介于一维 碳纳米管和二维石墨片之间. Tuinstra等 ${ }^{7}$ 计算了石 墨的振动模式, 利用所得的振动模式对石墨的拉曼 光谱 $G$ 峰做出了解释. 肖杨等 ${ }^{8}$ 通过五步旋转操作得 到了不同位置原子间的力常数矩阵, 对二维石墨片 的声子谱和振动模式密度进行了模拟计算.

分子振动模式的确定是进行分子振动光谱分 析与研究的基础. 利用群论对称操作特征标表可以 不求解复杂的久期方程而确定分子振动模式. 孙风 久等 ${ }^{9}$ 利用因子群分析法对方解石 $\left(\mathrm{CaCO}_{3}\right)$ 晶体的分 子振动模式进行了分析, 得到方解石晶体的具体振 动模式, 并明确指出了各振动模式的光谱特性. 彭 同江等 ${ }^{10}$ 运用因子群分析法对金云母和金云母-蛭 石矿物进行了振动模式分析, 成功预测了金云母和 金云母-蛭石结构的红外和拉曼光谱吸收带的数目.

目前, 关于石墨烯晶体分子振动模式的因子群 分析方法尚未见文献报道. 为了获得石墨烯晶体各 具体分子振动模式及其光谱特性, 本文运用因子群 分析法对石墨烯晶体振动模式进行群论分析, 并采
用基于密度泛函理论的从头算量子力学程序 CASTEP $^{11}$ 对石墨烯晶体进行分子振动光谱计算.

\section{2 晶体结构与布拉维原始晶胞的划分}

层状结构晶体中层内原子以较强的共价键结 合, 层与层之间以较弱的范德华键结合, 单质石墨 具有这种典型层状结构, 其结构单元层即为二维石 墨烯. 石墨烯结构中碳原子以六方形蜂窝点阵在二 维平面上有序排列(如图 1 所示), 其所属空间群为 P6/ mmm. 石墨烯的布拉维单胞中有 2 个等价的碳原 子(图 1 所示), 整个二维格子由这样的单胞周期性重 复排列构成. CASTEP 模块计算所采用的布拉维单 胞模型如图 2 所示.

\section{3 因子群分析}

\section{1 石墨烯布拉维晶胞碳原子的位置对称分析}

石墨烯具有六方二维结构, 点群为 $D_{6 h}$. 由图 2 石墨烯布拉维格子可见, 布拉维单胞中有两个等效 碳原子; 其碳原子的位置对称群为 $D_{3 h}$. 根据石墨烯 所属点群 $D_{6 h}$ 的不可约表示特征标表, ${ }^{12}$ 对石墨烯的 可约表示特征标进行计算, 结果列于表 1. 利用不可 约表示分解公式 ${ }^{10}$ 对石墨烯分子各简正振动模式进

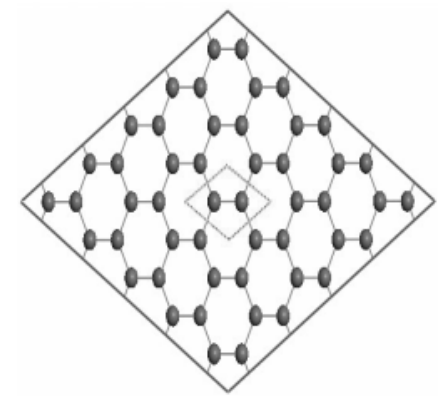

图 1 石墨烯的晶体结构

Fig.1 Crystal structure of graphene

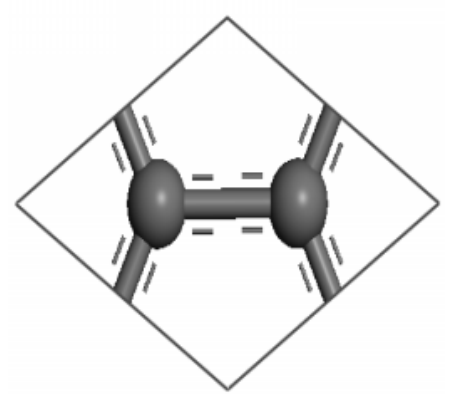

图 2 石墨烯的布拉维单胞

Fig.2 Bravais cell of graphene

表 1 石墨烯可约表示特征标计算表

Table 1 Characteristic indices of reducible representation of graphene

\begin{tabular}{|c|c|c|c|c|c|c|c|c|c|c|c|c|c|}
\hline \multicolumn{2}{|c|}{$D_{6 h}$} & $I^{\mathrm{a}}$ & $2 C_{6}{ }^{\mathrm{b}}$ & $2 C_{3}^{\mathrm{c}}$ & $C_{2}{ }^{\mathrm{d}}$ & $3 C_{2}^{\text {te }}$ & $3 C_{2}^{\prime \prime f f}$ & $i^{\mathrm{g}}$ & $2 S_{3}^{\mathrm{h}}$ & $2 S_{6}^{\mathrm{i}}$ & $\sigma_{h}^{\mathrm{j}}$ & $3 \sigma_{d}{ }^{\mathrm{k}}$ & $3 \sigma_{v}^{\prime}$ \\
\hline \multicolumn{2}{|c|}{$\theta^{\mathrm{m}}$} & 0 & 60 & 120 & 180 & 180 & 180 & 180 & 120 & 60 & 0 & 0 & 0 \\
\hline \multicolumn{2}{|c|}{$\cos \theta$} & 1 & $1 / 2$ & $-1 / 2$ & -1 & -1 & -1 & -1 & $-1 / 2$ & $1 / 2$ & 1 & 1 & 1 \\
\hline \multicolumn{2}{|c|}{$D_{3 h}$} & I & - & $2 C_{3}$ & - & $3 C_{2}^{\prime}$ & - & - & $2 S_{3}$ & - & $\sigma_{h}$ & - & $3 \sigma_{v}$ \\
\hline$N^{\mathrm{n}}=2$ & $N_{\mathrm{C}}{ }^{\circ}$ & 2 & 0 & 2 & 0 & 2 & 0 & 0 & 2 & 0 & 2 & 0 & 2 \\
\hline \multicolumn{2}{|c|}{$N$} & 2 & 0 & 2 & 0 & 2 & 0 & 0 & 2 & 0 & 2 & 0 & 2 \\
\hline \multicolumn{2}{|c|}{$\Gamma_{\mathrm{N}}$} & 6 & 0 & 0 & 0 & -2 & 0 & 0 & -4 & 0 & 2 & 0 & 2 \\
\hline \multicolumn{2}{|c|}{$\Gamma_{\mathrm{A}}$} & 3 & 2 & 0 & -1 & -1 & -1 & -3 & -2 & 0 & 1 & 1 & 1 \\
\hline \multicolumn{2}{|c|}{$\Gamma_{\mathrm{o}}$} & 3 & -2 & 0 & 1 & -1 & 1 & 3 & -2 & 0 & 1 & -1 & 1 \\
\hline
\end{tabular}

${ }^{\mathrm{a}}$ identical operation, ${ }^{\mathrm{b}}$ sixth-order rotational symmetry operation, ${ }^{\mathrm{c}}$ third-order rotational symmetry operation, ${ }^{\mathrm{d}}$ vertical second-order rotational symmetry operation, ${ }^{\mathrm{c}}$ diagonal second-order rotational symmetry operation, ${ }^{\mathrm{f}}$ horizontal second-order rotational symmetry operation, ${ }^{\mathrm{g}}$ inversion operation, ${ }^{\mathrm{h}}$ third-order rotational reflection operation, ${ }^{\mathrm{i}}$ six-order rotational reflection operation, ${ }^{\mathrm{j}}$ horizontal symmetric plane operation, ${ }^{\mathrm{k}}$ diagonal symmetric plane operation, ${ }^{1}$ vertical symmetric plane operation, ${ }^{\mathrm{m}}$ rotation angle, ${ }^{\mathrm{n}}$ number of atom, ${ }^{\circ}$ number of carbon atom 
行计算, 结果列于表 2 .

根据振动理论分析, 总模式 $\left(\Gamma_{\mathrm{N}}\right)$ 个数为 6 , 其中 声学模式 $\left(\Gamma_{\mathrm{A}}\right)$ 个数为 3 , 光学模式 $\left(\Gamma_{\mathrm{O}}\right)$ 个数为 3 . 由表 2 计算结果可知石墨烯在布里渊区中心 $q=0$ 处有 6 个简正模式, 即

总模式: $\Gamma_{\mathrm{N}}=B_{2 \mathrm{~g}}+E_{2 \mathrm{~g}}+A_{2 \mathrm{u}}+E_{1 \mathrm{u}}$

声学支: $\Gamma_{\mathrm{A}}=A_{2 \mathrm{u}}+E_{\text {lu }}$

光学支: $\Gamma_{\mathrm{0}}=B_{2 \mathrm{~g}}+E_{2 \mathrm{~g}}$

式中, $A_{2 \mathrm{u}}$ 和 $E_{1 \mathrm{u}}$ 表示为石墨烯平面内的声学模, $A_{2 \mathrm{u}}$ 是 碳原子移动方向垂直于石墨烯平面的声学模式 (ZA), $E_{1 \mathrm{u}}$ 为产生石墨烯平面内纵声学波(LA)和横声 学波(TA)两声学支声子谱的二重简并模式; $B_{2 g}$ 是碳 原子移动方向垂直于石墨烯平面的光学振动模式 $(\mathrm{ZO}) ; E_{2 \mathrm{~g}}$ 是平面内产生纵光学波(LO) 和横光学波 (TO)两光学支声子谱二重简并的光学振动模式. ${ }^{13}$

\section{2 石墨烯分子振动光谱活性分析}

在分子振动过程中, 只有分子电偶极矩发生变 化的振动模式才是红外活性的; 而极化率发生变化 的振动模式是拉曼活性的. ${ }^{14}$ 各对称类型的振动模 式活性列于表 2 .

红外吸收是因分子电偶极矩 $\mu$ 变化而产生的, 其分量 $\Gamma_{x} 、 \Gamma_{y} 、 \Gamma_{z}$ 所属不可约表示分别与 $x 、 y 、 z$ 相一 致, 从 $D_{6 h}$ 特征标表可得

$$
\Gamma_{\mu x} \rightarrow \Gamma_{x} \rightarrow E_{\mathrm{lu}}, \Gamma_{\mu y} \rightarrow \Gamma_{y} \rightarrow E_{\mathrm{lu}}, \Gamma_{\mu z} \rightarrow \Gamma_{z} \rightarrow A_{2 \mathrm{u}}
$$

故具有红外跃迁光谱活性的模式是 $A_{2 \mathrm{u}}$ 和 $E_{\mathrm{lu}}$, 且 $E_{\mathrm{lu}}$ 为二重简并模式. 由于 $A_{2 u}$ 和 $E_{1 \mathrm{u}}$ 振动模式在石墨烯 布里渊区中心处属于声学模式, 不与光作用, 因此 石墨烯无红外活性.

拉曼散射是由于感应偶极矩 $\left(\Gamma_{\mathrm{ind}}\right)$ 产生的, 与分 子的极化率 $(\alpha)$ 有关, 其各分量所属的不可约表示与
相应极化率张量分量的下标一致, 即

$$
\Gamma\left(\alpha_{x x}\right) \rightarrow \Gamma_{x x} \rightarrow E_{2 \mathrm{~g}}, \Gamma\left(\alpha_{y y}\right) \rightarrow \Gamma_{y y} \rightarrow E_{2 \mathrm{~g}}, \Gamma\left(\alpha_{z z}\right) \rightarrow \Gamma_{z z} \rightarrow A_{1 \mathrm{~g}}
$$

因此, 石墨烯具有拉曼光谱活性的振动模式是 $E_{2 \mathrm{~g}}$, 且 $E_{2 \mathrm{~g}}$ 为二重简并模式.

\section{4 模拟计算与验证分析}

利用 CASTEP 程序模块对建立的石墨烯布拉 维单胞模型(如图 2 所示)进行结构优化与频率计算. 平面波截断能量收玫为 $800 \mathrm{eV}$, 采用 $4 \times 4 \times 2$ 的 $k$ 点 抽样, 在布里渊区内进行倒易空间积分. 自洽精度 每个原子能量收玫值为 $5 \times 10^{-7} \mathrm{eV}$. 石墨烯布拉维单 胞中的原子数 $N=2$, 分子简正振动模式数为 6 , 采用 局域密度近似(LDA), ${ }^{15} \mathrm{CA}-\mathrm{PZ}$ 泛函可以对其振动频 率及相应的红外和拉曼光谱进行理论计算. 石墨烯 分子体系在正则坐标系统中的哈密顿形式 ${ }^{16}$ 为

$$
\mathbf{H}=\frac{1}{2} \sum_{i=1}^{6}\left(\frac{\mathrm{d} Q_{i}}{\mathrm{~d} t}\right)^{2}+\frac{1}{2} \sum_{i=1}^{6} k_{i} Q_{i}^{2}=\sum_{i=1}^{6} H_{i}
$$

其中, $Q$ 为石墨烯分子的正则坐标, $H_{i}$ 是第 $i$ 个振动 模式的哈密顿量, $k_{i}$ 是系数. 振动基态波函数为

$$
\psi\left(0_{1}, 0_{2}, \cdots, 0_{6}\right)=\prod_{i=1}^{6} \psi\left(0_{i}\right)
$$

该模型吸收能量后会有一个正则振动, 从基态跃迁 到第一激发态可以表示为

$$
\prod_{i=1}^{6} \psi_{i}(0) \rightarrow \Phi_{j}(1) \prod_{i \neq j}^{6} \Phi_{i}(0)
$$

在此模型下可以完成其理论计算. 经过计算后 所得的石墨烯的分子振动频率结果如表 3 所示.

理论计算所得单层石墨烯声子谱如图 3 所示, 对分子振动频率作图得到石墨烯拉曼光谱如图 4所 示.

第一性原理计算所得结果(表3)同因子群分析

表 2 石墨烯的振动模式及其分配与活性

Table 2 Vibrational mode, distribution and activity of graphene

\begin{tabular}{ccccc}
\hline Vibrational mode & Total mode & Acoustics mode & Optical mode & Vibrational active \\
\hline$A_{1 \mathrm{~g}}$ & 0 & 0 & 0 \\
$A_{2 \mathrm{~g}}$ & 0 & 0 & 0 \\
$B_{\mathrm{lg}}$ & 0 & 0 & 0 \\
$B_{2 \mathrm{~g}}$ & 1 & 0 & 1 & Raman active \\
$E_{\mathrm{lg}}$ & 0 & 0 & 0 & 1 \\
$E_{2 \mathrm{~g}}$ & 1 & 0 & 0 & 0 \\
$A_{1 \mathrm{u}}$ & 0 & 0 & 0 & \\
$A_{2 \mathrm{u}}$ & 1 & 1 & 0 & \\
$B_{1 \mathrm{u}}$ & 0 & 0 & 0 & \\
$B_{2 \mathrm{u}}$ & 0 & 0 & 0 & \\
$E_{1 \mathrm{u}}$ & 1 & 1 & 0 & \\
$E_{2 \mathrm{u}}$ & 0 & 0 & 0 & \\
\hline
\end{tabular}


表 3 单层石墨烯分子振动频率计算结果

Table 3 Calculated results of vibrational frequency of monolayer graphene

\begin{tabular}{ccccc}
\hline Vibrational mode & Degeneracy & Vibrational frequency $/ \mathrm{cm}^{-1}$ & Infrared active & Raman active \\
\hline$E_{\mathrm{lu}}$ & 2 & 0.0 & no & no \\
$A_{2 \mathrm{u}}$ & 1 & 0.0 & no & no \\
$B_{2 \mathrm{~g}}$ & 1 & 917.8 & no & no \\
$E_{2 \mathrm{~g}}$ & 2 & 1585.4 & no & yes \\
\hline
\end{tabular}

所得结果一致, 石墨烯布里渊区中心 $q=0$ 处只具有 $B_{2 g} 、 E_{2 g} 、 A_{2 u} 、 E_{1 \mathrm{u}}$ 四种分子振动模式. 在晶体分子振动 模式中声学模式与光学模式在布里渊区中心不同 之处在于: 声学模式的频率趋于 0 , 而光学模式的频 率趋于某一个定值. ${ }^{17}$ 结合表 3 和图 3 可知: $A_{2 \mathrm{u}}$ 和 $E_{1 \mathrm{u}}$ 分别对应石墨烯的 3 支声学模式, 在布里渊区中心 处 $A_{2 \mathrm{u}}$ 对应 ZA 声学支, $E_{\mathrm{lu}}$ 为二重简并声学模式, 当 结构对称性降低时分裂为 TA 和 LA 两声学支; $B_{2 \mathrm{~g}}$ 和 $E_{2 \mathrm{~g}}$ 分别对应石墨烯 3 支光学模式, 在布里渊区中心

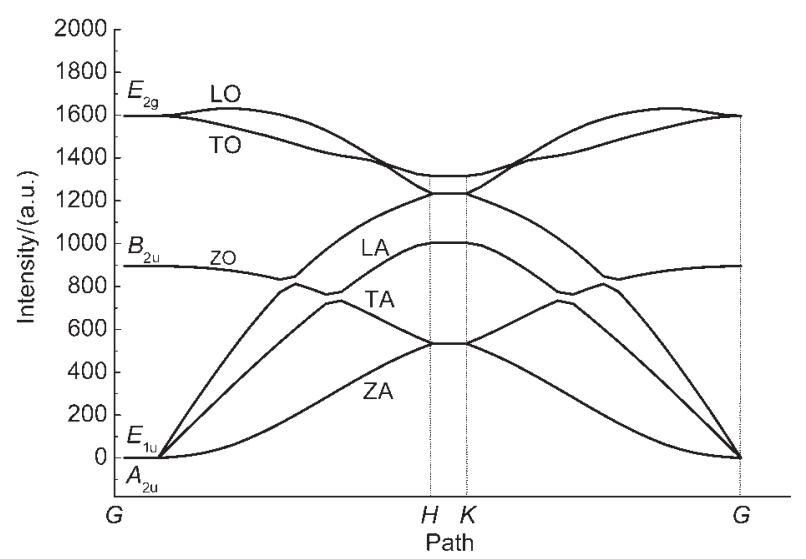

图 3 单层石墨烯声子谱图

Fig.3 Phonon spectra of monolayer graphene LO: longitudinal optical-phonon; TO: horizontal optical-phonon; ZO: $z$-axis direction of the optical-phonon; LA: longitudinal acoustic-phonon; TA: horizontal acoustic-phonon; ZA: $z$-axis direction of the acoustic-phonon

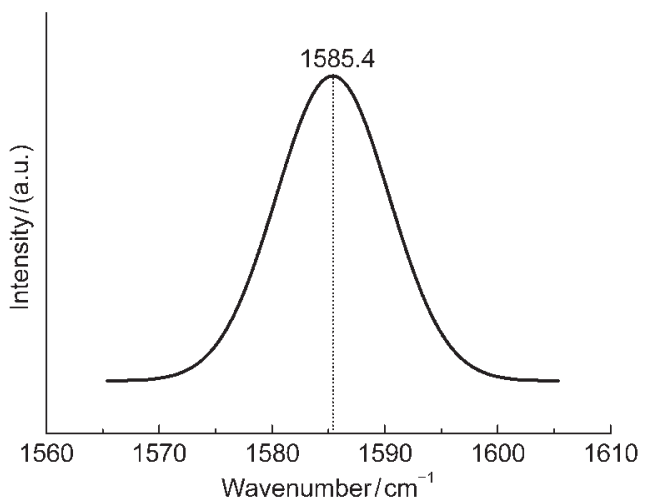

图 4 单层石墨烯计算拉曼光谱图

Fig.4 Computed Raman spectrum of monolayer graphene
处 $B_{2 g}$ 对应 $\mathrm{ZO}$ 光学支, $E_{2 \mathrm{~g}}$ 为二重简并光学模式, 当 结构对称性降低时分裂为 LO 和 TO 两光学支. 结合 图 3 和图 4 可以看出石墨烯拉曼活性由二重简并的 光学模式 $E_{2 g}$ 振动产生. 计算所得石墨烯 $E_{2 \mathrm{~g}}$ 振动频 率同实验一阶拉曼位移 $\left(1583 \mathrm{~cm}^{-1}\right)^{18-20}$ 相一致, 对应 理论石墨烯拉曼 $G$ 峰. 从表 3 中可以看出理论单层 石墨烯没有显示红外活性, 这同因子群分析所得结 论相吻合.

理论石墨烯拉曼光谱中除了 $1583 \mathrm{~cm}^{-1}$ 波数位 置的拉曼 $G$ 峰外, 在高波数区 $2700 \mathrm{~cm}^{-1}$ 位置还存在 一个 $2 D$ 峰. ${ }^{20} 2 D$ 峰为 $D$ 峰的倍频峰, 由双声子共振 拉曼散射产生. ${ }^{20,21}$ 在结晶程度较好的石墨烯拉曼光 谱中观察不到波数为 $1350 \mathrm{~cm}^{-1}$ 位置的 $D$ 峰, 因为 $D$ 峰的产生是伴随晶形缺陷而产生, $D$ 峰由呼吸模式 $A_{1 \mathrm{~g}}$ 引起, ${ }^{22}$ 只有当石墨烯晶面存在缺陷时, 石墨烯局 部对称性降低, 呼吸模式 $A_{1 \mathrm{~g}}$ 才表现拉曼活性, 因此 实验拉曼光谱图中 $D$ 峰又被称为“缺陷峰”.

单层石墨又叫理论石墨烯, 但是理论石墨烯的 红外活性与石墨相差较大. 石墨具有红外活性而理 论石墨烯没有红外活性, 原因是当高对称的石墨烯 组成低对称的石墨时, 使石墨烯的振动模式产生分 裂, 由非活性模式变为活性模式. 根据石墨烯振动 模式可分裂为一个高频异相振动和一个低频同相 振动理论, ${ }^{23}$ 当石墨烯 $E_{2 \mathrm{~g}}$ 光学模同相组合时产生一 个 $E_{2 \mathrm{~g}}$ 光学模, 表现为石墨在频率为 $1583 \mathrm{~cm}^{-1}$ 的拉

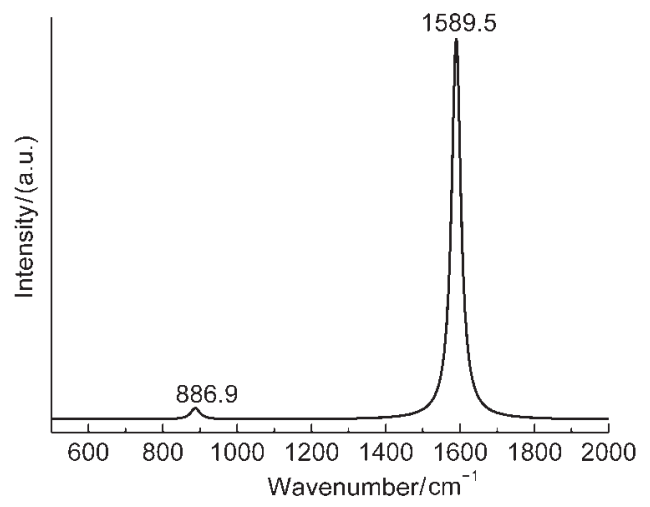

图 5 双层石墨烯二聚体计算红外光谱

Fig.5 Computed IR spectrum of double-layer graphene dimer 


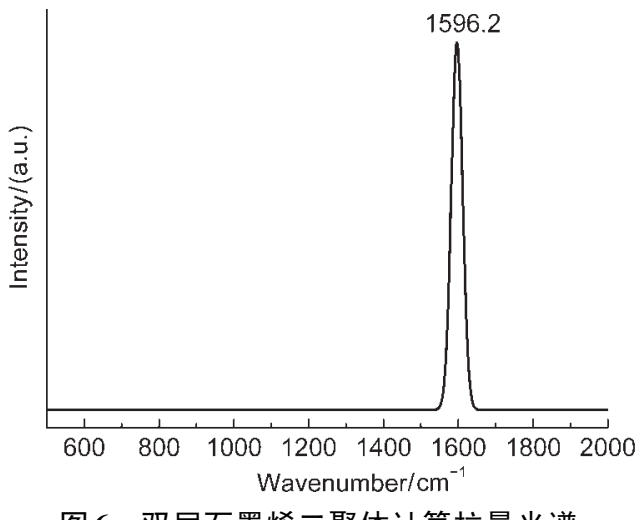

图 6 双层石墨烯二聚体计算拉曼光谱

Fig.6 Computed Raman spectrum of double-layer graphene dimer

曼活性振动; 当为异相组合时产生 $E_{\mathrm{lu}}$ 光学模, 表现 为石墨在频率为 $1583 \mathrm{~cm}^{-1}$ 的红外振动活性. 当石墨 烯 $B_{2 \mathrm{~g}}$ 光学模同相组合产生 $A_{2 \mathrm{u}}$, 表现为石墨在频率 为 $868.0 \mathrm{~cm}^{-1}$ 的红外振动活性; 当为异相组合时产 生 $B_{2 g}$ 模, 为石墨的非活性振动. 点群 $D_{6 h}$ 中的振动模 式 $A_{2 \mathrm{u}}$ 和 $E_{\mathrm{lu}}$ 是红外活性的, 但是理论石墨烯却不显 示红外活性, 因为 $A_{2 \mathrm{u}}$ 和 $E_{\mathrm{lu}}$ 振动模使碳-碳键在同方 向上做同位移平动, 属于声学模式, 没有产生偶极 距的净变化, 且声学模式是不与光作用的, 不产生 偶极距. ${ }^{24}$ 而石墨的两个红外吸收峰分别是由 $A_{2 \mathrm{u}}$ 和 $E_{1 \mathrm{u}}$ 两个振动模式引起的. 石墨共有 12 个简正模式, 声学模式由两个 $E_{1 \mathrm{u}}$ 和一个 $A_{2 \mathrm{u}}$ 模式构成, 另外两个 $E_{1 \mathrm{u}}$ 和一个 $A_{2 \mathrm{u}}$ 模式构成光学模中的 3 支, 使得石墨晶 体中碳-碳键振动在水平和坚直方向上有两个非对 称振动, 导致偶极距的变化, 因此石墨晶体具有两 个红外吸收峰. 这两个红外吸收峰被 Nemanich 等 ${ }^{25}$ 在红外测试实验中分别于 867.8 和 $1583 \mathrm{~cm}^{-1}$ 处观测 证实. 通过理论计算双层石墨烯二聚体的红外光谱 (如图 5 所示)和拉曼光谱(如图 6 所示)可知双层石墨 烯二聚体在波数为 886.9 和 $1589.5 \mathrm{~cm}^{-1}$ 位置处有两 个红外吸收峰, 分别与石墨 867.8 和 $1583.0 \mathrm{~cm}^{-1}$ 位 置处两个红外吸收峰相对应. 双层石墨烯二聚体 $1596.2 \mathrm{~cm}^{-1}$ 位置的拉曼峰由 $E_{2 \mathrm{~g}}$ 振动模产生, 对应石 墨 $1583.0 \mathrm{~cm}^{-1}$ 位置的拉曼 $G$ 峰.

\section{5 结 论}

(1) 利用因子群分析法确定了石墨烯布拉维单 胞的 6 个晶格振动模式, 其中包括 3 支声学模式和 3 支光学模式. 总模式 $\Gamma_{\mathrm{N}}=B_{2 \mathrm{~g}}+E_{2 \mathrm{~g}}+A_{2 \mathrm{u}}+E_{1 \mathrm{u}}$; 声学支 $\Gamma_{\mathrm{A}}=$ $A_{2 \mathrm{u}}+E_{1 \mathrm{u}}$; 光学支 $\Gamma_{\mathrm{o}}=B_{2 \mathrm{~g}}+E_{2 \mathrm{~g}}$, 其中 $B_{2 \mathrm{~g}}$ 是非活性振动模 式, $E_{2 \mathrm{~g}}$ 是拉曼活性振动模式, 且 $E_{1 \mathrm{lu}}$ 和 $E_{2 \mathrm{~g}}$ 分别为二重
简并的振动模式.

(2) 通过第一性原理对石墨烯分子振动频率、声 子谱及拉曼光谱计算分析可知石墨烯的一阶拉曼 活性是由 $E_{2 \mathrm{~g}}$ 光学振动模式引起. 计算所得石墨烯的 一阶拉曼活性位移在 $1585.0 \mathrm{~cm}^{-1}$ 与实验值 1583.0 $\mathrm{cm}^{-1}$ 相近, 对应石墨烯的拉曼 $G$ 峰.

(3) 点群 $D_{6 h}$ 中振动模式 $A_{2 u}$ 和 $E_{1 \mathrm{u}}$ 具有红外活性, 而石墨烯却不显示红外活性, 无红外吸收峰, 原因 是 $A_{2 \mathrm{u}}$ 和 $E_{1 \mathrm{u}}$ 振动模在石墨烯布里渊区中心位置属于 声学模式, 不与光作用, 无偶极距变化, 从而不显示 红外特性, 因此理论单层石墨烯只具有拉曼活性而 无红外活性.

致谢：感谢中国工程物理研究院姬广富研究员对本论文石 墨烯第一性原理计算的帮助以及 Materials Studio 计算软件 CASTEP 模块的支持.

\section{References}

(1) Novoselov, K. S.; Geim, A. K.; Morozov, S. V.; Zhang, Y.; Dubonos, S. V.; Grigorieva, I. V.; Firsov, A. A. Science 2004, 306,666

(2) Landau, L. D. Phys. Z. Sowjetunion 1937, 11, 26.

(3) Peierls, R. E. Ann Inst. Henri Poincare 1935, 5, 177.

(4) Geim, A. K.; Novoselov, K. S. Nat. Mat. 2007, 6, 183.

(5) Tse, W. K.; Das, S. S. Phys. Rev. Lett. 2007, 99, 236802.

(6) Liang, W.; Xiao, Y.; Ding, J. W. Acta Phys. Sin. 2008, 57 3714. [梁 维, 肖 杨, 丁建文. 物理学报, 2008, 57, 3714.]

(7) Tuinstra, F.; Koenig, J. L. J. Chem. Phys. 1970, 53, 1127.

(8) Xiao, Y.; Yan, X. H.; Cao, J. X.; Ding, J. W. Acta Phys. Sin. 2003, 7, 1720. [肖 杨, 颜晓红, 曹觉先, 丁建文. 物理学报, 2003, 7, 1720.]

(9) Sun, F. J.; Lou, D. H.; Li, L. J. Journal of Northeastern University (Natural Science) 2008, 29, 145. [孙凤久, 楼丹花, 李莉娟. 东北大学学报: 自然科学版, 2008, 29, 145.]

(10) Peng, T. J. Study on Crystal Chemistry of PhlogopiteVermiculite from Weili, XinJiang. Ph. D. Dissertation, China University of Geosciences, Beijing, 1993. [彭同江. 新疆尉犁 县且干布拉克蛭石矿金云母-蛭石间层矿物的晶体化学研究 [D]. 北京: 中国地质大学, 1993.]

(11) Segall, M. D.; Lindan, P. L. D.; Probert, M. J.; Pickard, C. J.; Hasnip, P. J.; Clark, S. J.; Payne, M. C. J. Phys.: Condens. Matter 2002, 14, 2717

(12) Lin, M. H. Concise Guide of Quantum Chemistry; Chemical Industry Press: Beijing, 2005; p 274. [林梦海. 量子化学简明 教程. 北京: 化学工业出版社, 2005: 274.]

(13) Falkovsky, L. A. Phys. Lett. A 2008, 372, 5191.

(14) Yang, X. G.; Wu, Q. L. Raman Spectroscopy Analysis and Application; National Defence Industry Press: Beijing, 2008; pp 
11-12. [杨序纲, 吴琪琳. 拉曼光谱的分析与应用. 北京: 国 防工业出版社, 2008: 11-12.]

(15) Vosko, S. J.; Wilk, L.; Nusair, M. Can. J. Phys. 1980, 58, 1200.

(16) Zhang, X. M.; Wang, L. G.; Li, Y. J. At. Mol. Phys. 2008, 25, 755. [张秀梅, 王利光, 李 勇. 原子与分子物理学报, 2008, 25,755 .]

(17) Huang, K. Solid State Physics; Higher Education Press: Beijing, 1988; pp 92-103; adapted by Han, R. Q. [黄 昆. 固体物理 学. 韩汝琦, 改编. 北京: 高等教育出版社, 1988: 92-103.]

(18) Balandin, A. A.; Ghosh, S.; Bao, W. Z.; Calizo, I.; Teweldebrhan, D.; Miao, F.; Lau, C. N. Nano Lett. 2008, 8, 903.

(19) Yang, Y. H.; Sun, H. J.; Peng, T. J.; Huang, Q. Acta Phys. -Chim. Sin. 2011, 27, 740. [杨勇辉, 孙红娟, 彭同江, 黄 桥. 物理化 学学报, 2011, 27, 740.]
(20) Ferrari, A. C.; Meyer, J. C.; Scardaci, V.; Casiraghi, C.; Lazzeri, M.; Mauri, F.; Piscanec, S.; Jiang, D; Novoselov, K. S.; Roth, S.; Geim, A. K. Phys. Rev. Lett. 2006, 97, 187401.

(21) Malarda, L. M.; Pimenta, M. A.; Dresselhaus, G.; Dresselhaus, M. S. Phys. Rep. 2009, 473, 54.

(22) Ferrari, A. C.; Robertson, J. Phys. Rev. B 2000, 61, 14098.

(23) Stephanie, R.; Christian, T. Phil. Trans. R. Soc. Lond. A 2004, 362, 2273.

(24) Wu, G. Z. Raman Spectroscopy: An Intensity Approach; Science Press: Beijing, 2007; pp 61-62. [吴国桢. 拉曼谱学: 峰强中 的信息. 北京: 科学出版社, 2007: 61-62.]

(25) Nemanich, R. J.; Lucovsky, G.; Solin, S. A. Mater. Sci. Eng. 1977, 31, 157. 